\title{
Architectures for smart end-user services in the power grid
}

\author{
Tom Verschueren, Wouter Haerick, Kevin Mets, \\ Chris Develder, Filip De Turck \\ Ghent University - IBBT, \\ Dept. of Information Technology - IBCN, Ghent, Belgium \\ Email: tom.verschueren@intec.ugent.be
}

\author{
Thierry Pollet \\ Alcatel-Lucent \\ Research and innovation \\ Antwerp, Belgium \\ Email: thierry.pollet@alcatel-lucent.com
}

\begin{abstract}
The increase of distributed renewable electricity generators, such as solar cells and wind turbines, requires a new energy management system. These distributed generators introduce bidirectional energy flows in the low-voltage power grid, requiring novel coordination mechanisms to balance local supply and demand. Closed solutions exist for energy management on the level of individual homes. However, no service architectures have been defined that allow the growing number of end-users to interact with the other power consumers and generators and to get involved in more rational energy consumption patterns using intuitive applications. We therefore present a common service architecture that allows houses with renewable energy generation and smart energy devices to plug into a distributed energy management system, integrated with the public power grid. Next to the technical details, we focus on the usability aspects of the end-user applications in order to contribute to high service adoption and optimal user involvement.

The presented architecture facilitates end-users to reduce net energy consumption, enables power grid providers to better balance supply and demand, and allows new actors to join with new services. We present a novel simulator that allows to evaluate both the power grid and data communication aspects, and illustrate a $22 \%$ reduction of the peak load by deploying a central coordinator inside the home gateway of an end-user.

Index Terms-smart power grid, rational energy consumption, end-user services
\end{abstract}

\section{INTRODUCTION}

The power grid is moving away from the current centralized power generation paradigm. With governments promoting local renewable power generation at residential sites, distributed power generation is gaining in popularity. Environmental concerns are the driving force for the replacement of traditional energy sources by green alternatives. Additionally, a large increase in electricity demand is expected as traditional energy sources are getting replaced by electricity. This is the case for cars, for which all major car manufacturers propose the plugin hybrid electrical vehicle (PHEV) as a solution for a more environmentally friendly car.

The increasing usage of renewable energy sources will be highly dependant on the roll out of a smart energy grid. The smart grid, consisting of an ICT architecture that allows to control the complexity of the future power grid, needs to be able to deal with unpredictable, distributed power generation and consumption. Typical issues of the future power grid are voltage and frequency instabilities as a result of local power generation, power security issues resulting from bidirectional energy flows and less predictable demand supply matching given the nature of renewable energy sources and the flexibility of loads that are shifted in time. A control plane is required that ensures availability, efficiency and low emissions. The benefits of the smart grid are not limited to the power distributors but reach both industrial and residential customers as well. By deploying the proper control mechanisms, the power distributor can save money by avoided investments for additional capacity. The industrial and residential customers benefit from green, locally produced power and lower energy bills by automated shifting of flexible loads towards cheaper time windows. To enjoy these benefits, an integrated ICT network for controlling (distributed) energy resources is required.

The ICT network and services provide a complete, real-time control of energy consumption and production in the power grid. This allows for the implementation of advanced demand response services such as peak shaving and load flattening [1]. Not only are high power demands steered towards high production times, the locality of the distributed generators can be exploited. By moving generation capabilities closer to the consumer, transmission costs and losses can be avoided.

Additional services in the smart grid aimed at the end-user

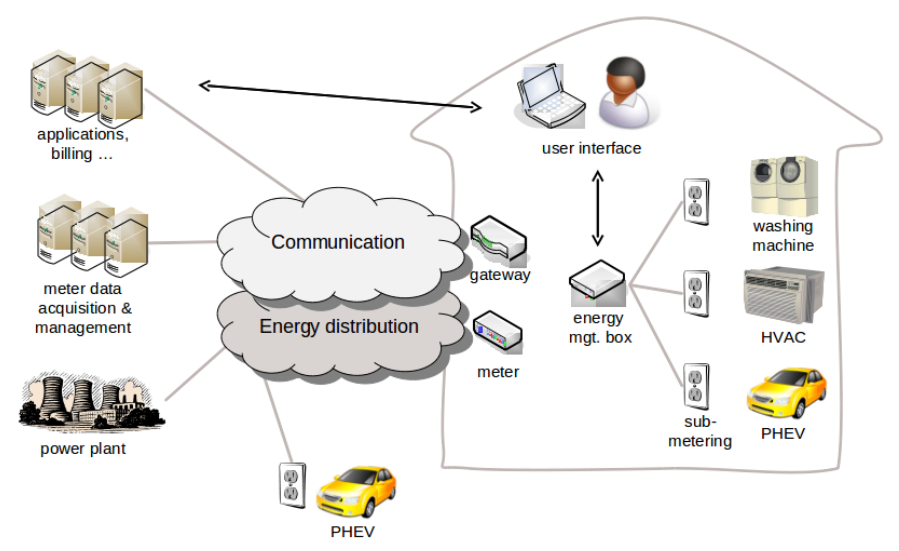

Fig. 1: Smart grid actors and components: The power grid is augmented with a communication interconnecting energy management boxes with service providers. 
can be deployed. Such services may lower the cost of energy by shifting loads, but could also involve the customer in the reduction of his energy consumption. By making the enduser aware of his energy consumption pattern and behavior, giving detailed (real-time) information on consumption, energy can be saved. Such a service could be provided by a home energy management box, installed at the customers premises and managed by a service provider. Additional smart services, also installed in the home-box, can take a more active role for example by using dynamic load shifting techniques to move controllable device to low energy cost times.

In Figure 1, we illustrate the actors and components involved in smart grid end-user services. In the home, the energy management box hosts local intelligence (local services) and is connected to a multitude of smart devices: smart digital meter, a smart washing machine, a remotely controlled HVAC, a PHEV charging station, etc. To achieve optimal energy efficiency, the home energy box is connected through a communication network to external service providers that may offer additional intelligence. Additionally, service providers may offer supporting services such as billing applications, device scheduling services and realtime brokering services. In Figure 1, the plug-in electrical vehicle is assumed to recharge both at home and at public charging infrastructures.

The remainder of this paper is structured as follows: in Section II we give an overview of the current state-of-the-art in smart energy home protocols and control services. Subsequently, a number of smart energy use cases are presented. In Section IV we present a smart grid service architecture that allows for dynamic deployment of new end-user services. Next, we present a novel smart grid simulator in Section $\mathrm{V}$ that also models the ICT control plane, and present energy savings by shifting energy consumers in time. Final conclusions are summarized in Section VI.

\section{STATE-OF-THE-ART IN SMART ENERGY HOME ARCHITECTURES}

\section{A. Smart metering standardization efforts in Europe}

Whereas NIST [2] is in charge of standardization of the smart power grid in the United States, the European Commission has issued a mandate $M / 441$ to the three European standardization organisations CEN [3], CENELEC [4] and ETSI [5] to define an open architecture for utility meters and services. This mandate covers smart meter functionalities and communication for usage of electricity, gas, heat and water applications. Today, there are about 110 applicable technical standards available in the field of smart metering. However, no standard details an open architecture that covers the full application range. The mandate should ensure interoperability of technologies and applications within the European market.

The envisioned open architecture positions the smart meter gateway as central device in the home. This gateway could be part of any of the digital meters (electricity, gas, water, heat) or deployed as a separate device. Interfaces need to be defined to specify the communication with the in-home meters and home automation services. Additionally, an open interface is required to connect with a public network of service providers, offering two types of services:

- Technical services refer to those services that help to control the electrical grid and include smart grid stability services, smart grid security services, demand side management, etc.

- Commercial services are offered to the end-user and may comprise billing applications, tarif alerts, pre-payment systems, PHEV charging services, energy aggregator services, etc.

\section{B. Smart energy end-user services}

In today's market there are already a number of enduser services available. These services typically provide an energy dashbord that gives detailed monitoring information on residential energy usage. We distinguish:

- Local in-home solutions, which have no connection to a public communication network;

- On-line solutions, where the energy dashboard is connected to a service provider in the public communication network.

Google's PowerMeter and Greenbox's CustomerIQ are two examples that provide an online power monitoring website. Other solutions, such as Onzo's "Smart energy kit" and GEO's Home Energy Hub, make use of displays that are installed in the residence.

Both types of solutions merely provide real time information on total consumption cost and the source of the energy usage. Some of the solutions allow to switch off devices according to a given schedule. All these solutions however lack control algorithms to steer devices in an automated way, in response to real-time information on local energy production, energy tarifs and flexibility of energy needs. In the remainder of this paper, we will therefore focus on the control plane aspects of emerging end-user services in the power grid.

\section{SMART ENERGY USE CASES}

\section{A. Smart buildings and homes}

There exist various degrees to which the energy flow control plane affects the end-user environment. Traditionally, the enduser had no interaction with the flows in the control plane. He was merely an energy consumer whose consumption pattern was determined by his consumption behavior. This pattern was modeled by means of (synthetic) load profiles. New smart energy use cases arise that impact both the control plane and the energy plane. Two smart energy use cases are:

- Demand response services: Demand Side Management are energy applications that impact the energy flow towards the end-user endpoints. For these applications control was originally limited to interaction with the distribution network infrastructure. With the introduction of smart metering, this end-user consumption can now possibly be controlled at energy access point level. Smart meters with connect/disconnect features allow to disconnect homes from the grid, in case of a security issues. 


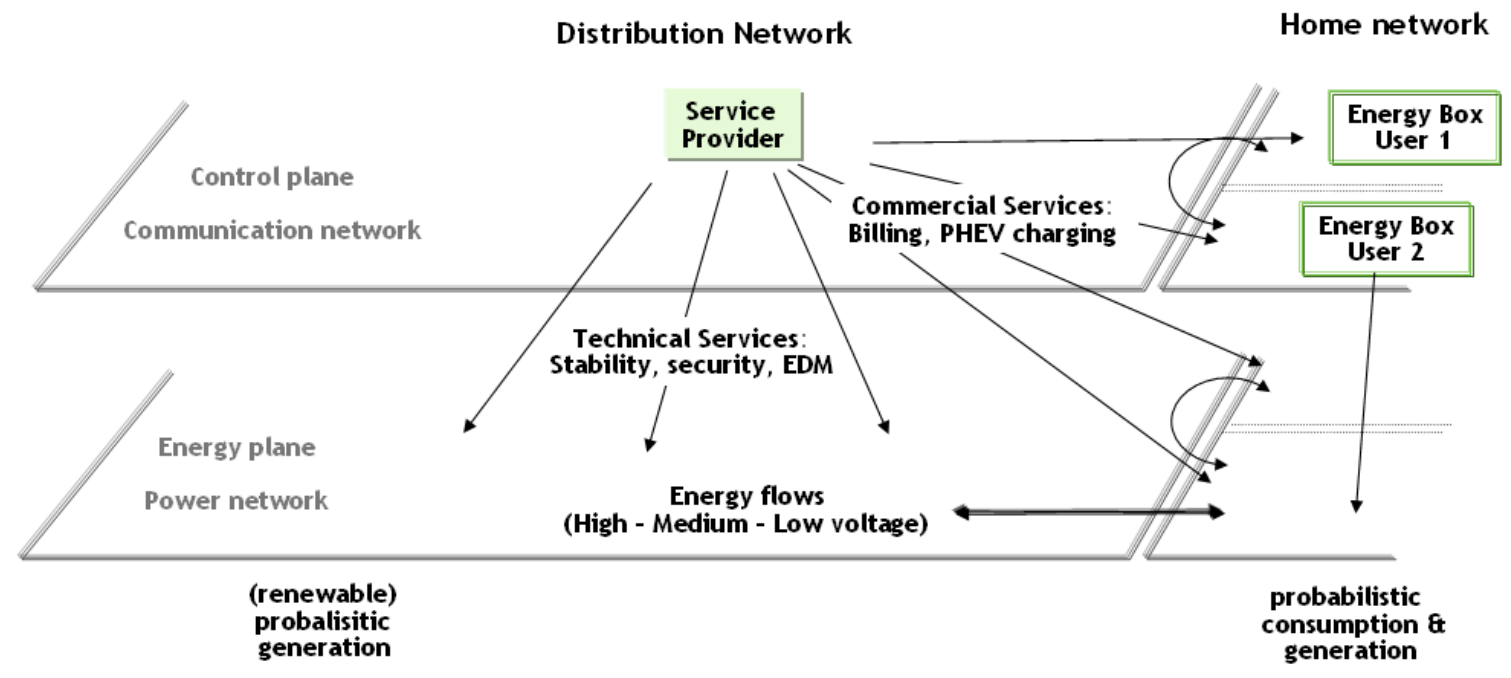

Fig. 2: Control plane and energy plane for emerging end-user services.

Demand Response programs encourage end-users to reduce their energy consumption in particular during peak loads. This can be achieved by extending the control plane into the home as to curtail the end-users energy consumption by controlling appliances (Figure 2). It is to be noted that, however, in some cases control is achieved at the commercial level, impacting directly the end-user pattern for instance as a response to various pricing alerts.

- Micro energy generation services: With the advent of micro energy generation and local storage, the control plane is to be further advanced as to handle bidirectional energy flows. With (micro) power consumption, generation and storage possibly becoming highly distributed, alternatives to centralized control are being proposed. A local community can be established where each of the households interact and trade energy with each other, in a peer-to-peer communication mode. Alternatively, a group of households could aggregate its renewable energy sources into a virtual power-plant and offer the resulting power to local enterprises.

\section{B. Adoption of end-user services}

Both use cases offer value for the end-user. The demand response service allows lowering the energy cost by shifting flexible energy loads, while the micro energy generation service allows maximum revenue from local generation. However, it is not clear if a majority of the end-users are willing to adopt these service in the coming years. A social study is necessary, evaluating the energy-aware behavior of the different categories of end-users. Some will adopt the load shifting service for the financial benefit, others will be reluctant to give up their comfort. Also, some adopters may prefer active involvement through displays and input devices while others may choose for a completely automated solution. The social study should reveal particular preferences from endusers. These preferences should be considered carefully while designing the end-user interface and the service interactions. By focusing on the end-user during the design of each of the service features, the resulting service will differentiate against solutions that merely implement the technical interfaces of standardization bodies.

\section{Simulation of business cases with dynamic grid fees}

Additionally, the roll-out of a smart power grid-and in particular the ICT service architecture of the smart power gridwill require large investments that may outweight the benefits for the different stakeholders. For the power grid operator, the cabling investments related to the increased line capacity needed for bidirectional energy flows may become obsolete if smart control algorithms succeed to shave peak loads. Business case simulations will be required to evaluate this trade-off, and to identify the savings for each of the stakeholders in the value chain.

Also, for the micro energy generation service the business case may become negative if the power grid operator starts charging grid fees-this is the fee associated with the transport of energy-to redistribute locally generated power. Scenarios need to be evaluated that consider dynamic pricing for the power as well as for the transport of the power (dynamic grid fee). The outcome of the simulations, may be of particular interest of the regulatory bodies that may need to define a regulatory context for dynamic grid fees.

\section{ENERGY SERVICES ARCHITECTURES}

To allow the interaction of houses with renewable energy generation and smart energy devices with the power grid, a pluggable energy services architecture needs to be designed. Other smart grid architectures have been proposed [6], [7]. The algorithm and architecture described in [7] provides a distributed control system for the electrical power structure. The micro grid management system [6], is an agent based system for the management of generation and storage devices in the 


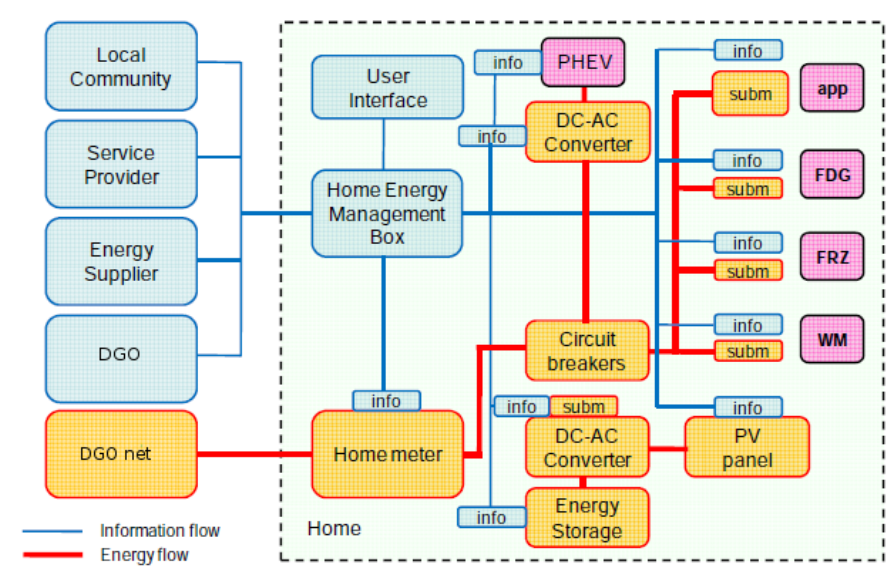

Fig. 3: High level component architecture of the power grid (energy flows) and the ICT grid (information flow).

low voltage grid. Both architectures lack support for remote deployment of new services. The goal of this architecture is to define an architecture which is both flexible and scalable to allow the creation of a wide range of use cases, both in the power distribution network and in the in-house network. Additionally, central as well as distributed control strategies should be supported. The envisioned architecture allows for the remote deployment of smart energy end-user applications and control algorithms that manage renewable energy sources and provide flexible end-user services such as billing processes, PHEV charging coordination, dynamic load shifting of smart devices, etc.

\section{A. The need for controlling entities}

The current electrical utility power systems were not designed to support active power generation at the distribution level, but assumed central power generation system. Distributed residential generators, such as photovoltaic (PV) panels, are an unpredictable source of energy and can be the cause of voltage fluctuations. In today's power grid, PV panels detecting such fluctuations will protect the grid by switching off themselves. As the current power grid is not designed for bi-directional power flow, security is another issue to be addressed. The problem is that electrical surge protectors are designed to isolate a segment of the grid when power instabilities occur in the distribution grid, they are not designed to handle the distributed generators. To support the benefits of distributed generators, while avoiding negative impacts on the grid's stability and security, a control system has to be created.

This control system needs to be able to interact on all levels of the power grid, e.g.: device level, house level, low voltage level and virtual aggregator level. Modules in higher levels are used to aggregate information from, and delegate control to the lower level modules. This allows, for example, stability control algorithms to have fine grained control of specific devices, while higher level abstractions provide support for multi-house optimization services

\section{B. Smart Grid Service architecture}

Figure 3 depicts the components of our Smart Grid Service architecture, that comprises a power grid and ICT grid. Communication between the ICT components can be provided by a mix of different physical media communication protocols such as Zigbee, power line communication, WiFi and Ethernet.

As shown in Figure 3, each of the residential appliances, such as the refrigerator (FDG), the freezer (FRZ) and the washing machine (WM), has been provided with a submeter (subm) and an ICT enabled control module (info). The submeters provide detailed power usage monitoring support and are connected to the circuit breaker. More advanced systems such as a PHEV + DC-AC converter and solar PV panels are also supported by the architecture. If these devices do not have a built-in submeter or ICT control module, these modules can be added separately. All ICT modules are connected with the Home Energy Management Box.

The Home Energy Management Box is the centralized component in the in-house energy management, and resides at the consumers premises. This Home-box can house smartapplications that control and communicate with the local devices. An external communication link is also provided, which can be used for the communication with remote systems of the power grid operator or of other service providers.

The Home Energy Management Box plays a major role in making consumers more aware, and thereby stimulating a change of their energy consumption. Information such as realtime consumption costs or comparisons with similar profile houses can be provided through an end-user GUI to the consumer. This GUI can be made available using different media, such as an interactive touchscreen, digital TV, PC or a mobile device.

The ICT grid allows for service providers to deploy (internet-based) services, such as a real time pricing service that informs customers of changes in the energy price, or demand response services that actively control smart devices. The distribution grid operator (DGO) and energy suppliers are also connected to the ICT network, allowing for flexible billing services.

Virtual aggregators are used to (virtually) group consumers in a local community based on certain criteria, such as their consumption profile, their flexibility in device shifting, etc. An automated segmentation and classification method is described in [8]. The aggregated group of consumers provides extra flexibility compared to a single consumer. This provides greater optimization capabilities for smart grid services.

Figure 4 shows how service components can be deployed on all levels of the smart grid. Through remote management functionality, service components can be distributed across internet-based servers, substations, the Home Energy Management Box and smart devices.

\section{Service interactions}

In this section we describe two scenarios to illustrate the service interactions in our Smart Grid Service architecture. The first scenario offers an automated demand response technique, 


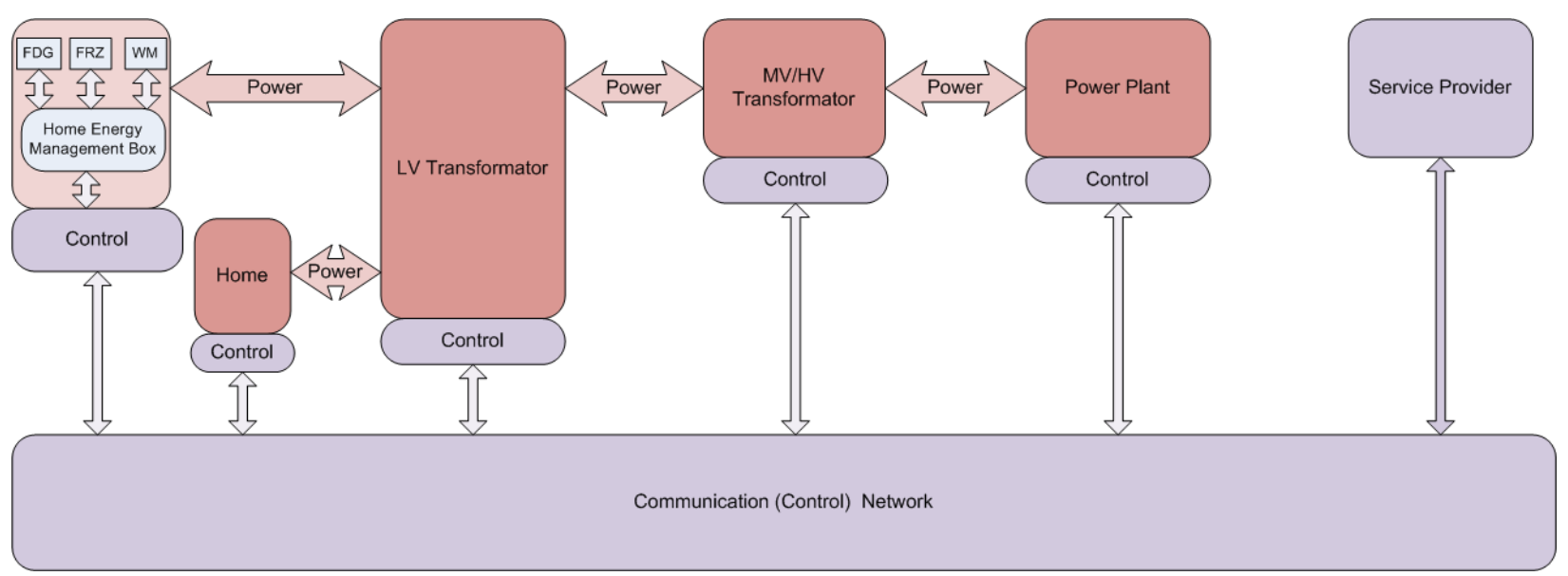

Fig. 5: Message based smart grid simulator with power grid components and control components

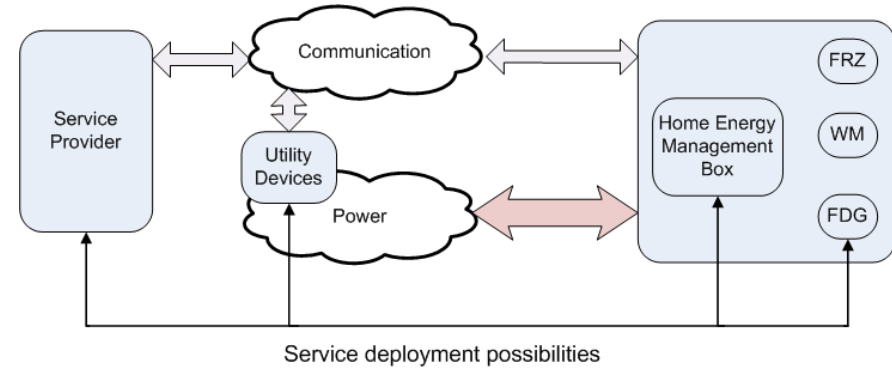

Fig. 4: Service components can be deployed on different levels.

and uses a load shifting algorithm to manage consumption of a set of devices. The goal of this service is to reduce peak loads in the distribution network. End-users can be offered incentives to join this service and have their devices externally managed. The load shifting service, which considers an entire group of households and devices, allows for an optimal result compared to managing all end-users separately. The end-users are grouped in local communities, acting as virtual aggregators. The grouping is based on selection criteria, such as the consumption profiles of the end-user, the available generation capacities, etc. The local community can be joined through the GUI offered by the home energy management box. Once joined, information on smart devices and generation capacity will be shared with the local community. The load shifting service interacts with the local community, which provides aggregated information on consumption and generation devices of all of its members. With this information, the load shifting service makes an optimal consumption and generation planning. This planning is delegated to all members by to the local community. Each home energy management box configures its local devices upon receiving their individual device planning.

A second scenario deals with the roll-out of PHEVs. As there is no control system available, each PHEV charges autonomously. This uncontrolled charging of all PHEVs can generate important power strains on the power grid [9], [10] . To handle this problem, a centralized charging coordination service can be created. End-users can again subscribe to this charging coordination service through the home energy management box. Once the service is deployed on the home box, this box can control and optimize the charging of each PHEV that plugs into the home power network. This service is then able to control when and at which rate each of the PHEVs charges.

\section{Simulation OF CONTROLling ALGORITHMS}

To evaluate various smart grid control algorithms, they need to be tested in different scenarios. These scenarios can vary in a large degree, such as in the used physical media communication protocols (Zigbee, PLC, ...), failure scenarios of both the power and ICT grid, scalability tests ranging from one house to an entire low-voltage grid, etc. As it is costly to create such a controllable infrastructure and impossible to use the existing power grid, the need for a simulator arises.

A smart grid simulator needs to be both flexible enough to support the large range of scenarios, as mentioned above, and scalable to support the simulation of a large number of homes and smart devices.

\section{A. Smart grid data simulator}

A number of simulators in the field of power system engineering exist, packages such as NEPLAN[11] and PSS NETOMAC \& SINCAL[12] provide professional network planning tools. Well known simulators for communication systems include NS-2[13], OPNET[14] and QualNET[15], they all provide a platform for large scale network simulations. The listed simulators all provide domain specific tools, for providing interaction between both the communication and power system, an additional framework needs to be provided.

The smart grid simulator presented in this paper provides such a framework. The framework is implemented in OMNeT++ [16] , which is an open, extensible, modular, component-based C++ simulation library and framework. Many domain-specific simulation models are already freely 
available, and can be easily integrated in new models. One of these models is the OMNeT++ INET framework [17]. This is an open-source communication networks simulation package and contains models for several communication protocols.

Two types of components play a major role in the smart grid simulator: the power grid and ICT grid control components. Simulation of the power grid is achieved by the power grid components. Programming interfaces for common power grid components, such as generators and transformers, have been created. Through these interfaces information related to the electrical properties of the components can be accessed. Different implementations of these interfaces can be provided, depending on the level of detail the electrical component has to be simulated. This provides a library of interfacebased components, with different degrees of complexity. When running a simulation, we can choose how deep we want to simulate the power grid by choosing the matching interface implementations.

Communication and control logic is provided by the ICT grid components, for which we again provide the required interfaces. The ICT components are responsible for providing the implementation of communication protocols (both physical and application), service applications, end-user applications, etc. Communication protocols can be modelled based on the available INET framework, offering a detailed simulation of the communication network. ICT components can be loosely coupled with power grid components to form a smart device, e.g. a controllable or intelligent electrical device. These devices can represent normal residential devices such as TVs, dryers or more complex devices like PHEVs and renewable energy generators. The ICT component can provide intelligence to the device allowing for autonomous decisions (e.g. dynamic device shifting or automatic meter updates) or it can simply act as a communication interface to the device, allowing the device to be controlled by external services.

In the simulator, all components use a message based communication system. A simple topology consisting of two houses connected to the low voltage power grid is shown in Figure 5. The low voltage transformer itself is connected to the medium/high voltage power grid, which receives power from a power plant. Services can be deployed by the service provider through the communication network.

A high level overview of the simulator usage is depicted in Figure 6. A simulation scenario is defined by two OMNeT++ configuration files: a file describing the topology of the network and an INI file configuring all parameters of the simulated components. Parameters include user profiles, consumption profiles and scenario descriptions. The topology file is written in the Network Description (NED) language, which is part of the OMNeT++ simulator. It is a feature rich language that allows great flexibility in the creation of simulation networks. The parameters described in the INI file have access to the statistical tools of OMNeT++ that allows the execution of a large set of random simulations.

First results of the simulator are given based on the automated demand response scenario described in section IV-C.

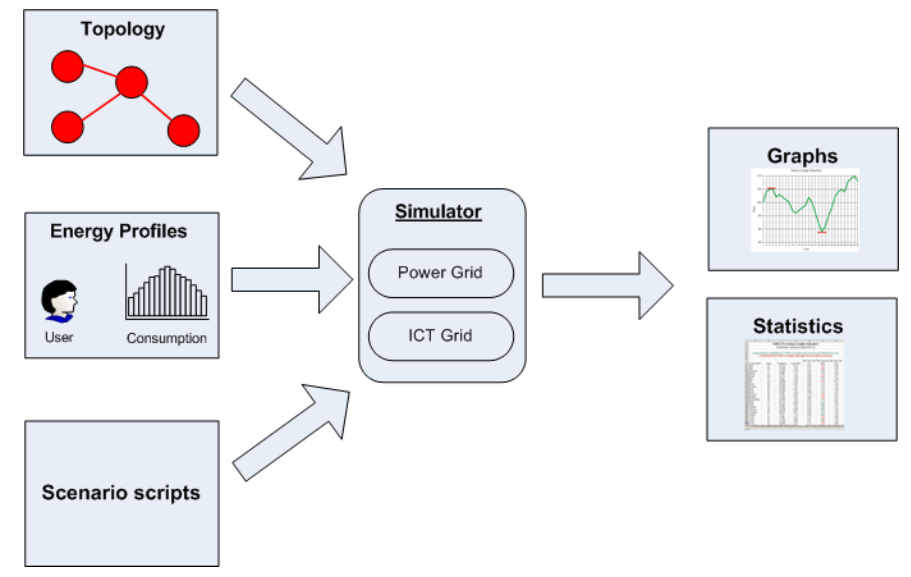

Fig. 6: A simulation run is defined by a topology, energy profiles and a scenario script.

We present a case where the usage of a set of residential devices(WM, DR \& DW) are planned. When no intelligent coordinator is presented, devices can start consuming during high energy consuming moments, causing higher peaks in the end-user's energy usage profile. Such peaks can cause large fluctuations in the power grid, requiring fast adaptable but less efficient peaking power plants. Field tests [18] have shown that shifting of residential devices can lead to significant peak reductions.

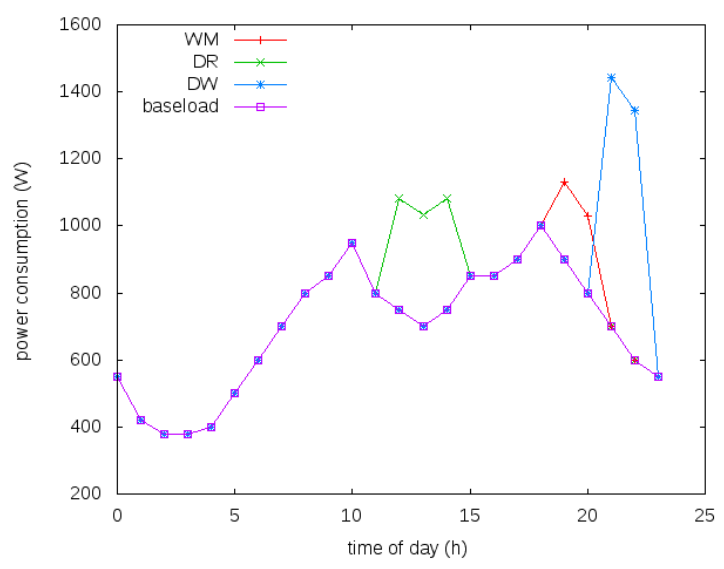

Fig. 7: Power consumption of a household with no load shifting

A central coordinator can move device usage away from peak moments, resulting in a smoother energy usage pattern. By analysing the historical energy usage, low consumption times are identified, to which shiftable devices are planned. The algorithm has as input the load and usage profiles of the shiftable devices (type, shifting flexibility, relation to other devices, etc.) and historical and future predictions of the local energy consumption and generation. Historical energy usage data is provided by Synthetic Load Profiles, provided by the Flemish Regulation Entity for the Electricity and Gas market (VREG)[19]. 
In Figures 7 and 8, the influence of load shifting on the energy consumption profile is shown. The graphs show the baseload, based on historical energy usage, completed with the energy usage of three shiftable devices. Figure 8 depicts the resulting power usage when the load shifting service is used and indicates a reduced peak load. For this specific case, peak loads could be reduced by $22 \%$.

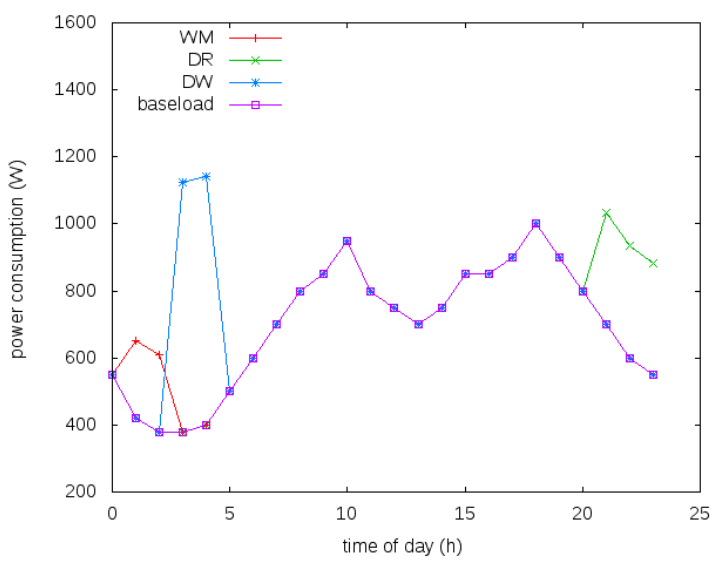

Fig. 8: Power consumption of a household with centrally coordinated load shifting.

\section{CONCLUSIONS}

In this paper we have presented a service architecture that integrates with the power grid, both in the power distribution network and the in-house power network. The architecture allows end-users with renewable energy production (wind, solar) and with shiftable loads to get involved in more rational energy consumption patterns, and to participate in the local balancing of energy demand and supply.

The architecture differs form other state-of-the-art architectures in that it offers a fully integrated control system, that allows the deployment of external services and control of all smart devices that are managed by the home energy management box. Local communities can be created by grouping end-users based on certain criteria. The presented service architecture not only allows end-users with renewable endusers to interact, but also supports interaction with new players in the value chain, such as energy trading services or energy brokers or aggregators. Within the scope of the IWT SmartE project, consumer-oriented services will be deployed in the presented service architecture and evaluated on their usability, the ability to save energy and new business models for these services.

A simulator has been designed that allows the evaluation of controlling entities deployed in the smart grid service architecture. The results from a load shifting simulation show a peak load reduction of $22 \%$.

\section{ACKNOWLEDGMENT}

Work presented in this paper has been supported by the Flemish Government through the LINEAR project (through
IWT) and the project SmartE (an IBBT ICON project). K. Mets would like to thank the Institute for the Promotion of Innovation by Science and Technology in Flanders (IWT) for financial support through his Ph. D. grant. C. Develder thanks the Research Foundation - Flanders (FWO - Vlaanderen) for his post-doctoral fellowship.

\section{REFERENCES}

[1] A. R. Severin Borenstein, Michael Jaske, "Dynamic pricing, advanced metering, and demand response in electricity markets." UC Berkeley: Center for the Study of Energy Markets, 2002. [Online]. Available: http://escholarship.org/uc/item/11w8d6m4

[2] NIST, "National institute of standards and technology," Jan. 2010. [Online]. Available: http://www.nist.gov/

[3] CEN, "the european committee for standardization," Jan. 2010. [Online]. Available: http://www.cen.eu/cenorm/

[4] CENELEC, "European committee for electrotechnical standardization," Jan. 2010. [Online]. Available: http://www.cenelec.eu/

[5] ETSI, "European telecommunications standards institute," Jan. 2010. [Online]. Available: http://www.etsi.org/

[6] J. Oyarzabal, J. Jimeno, J. Ruela, A. Engler, and C. Hardt, "Agent based micro grid management system," in Future Power Systems, 2005 International Conference on, Nov. 2005.

[7] J. Van de Vyver, G. Deconinck, and R. Belmans, "The need for a distributed algorithm for control of the electrical power infrastructure," in Computational Intelligence for Measurement Systems and Applications, 2003. CIMSA '03. 2003 IEEE International Symposium on, July 2003, pp. 211-215.

[8] S. Verdu, M. Garcia, F. Franco, N. Encinas, A. Marin, A. Molina, and E. Lazaro, "Characterization and identification of electrical customers through the use of self-organizing maps and daily load parameters," in Power Systems Conference and Exposition, 2004. IEEE PES, Oct. 2004, pp. 899-906 vol.2.

[9] D. J. Clement K., Haesen E., "The impact of uncontrolled and controlled charging of plug-in hybrid electric vehicles on the distribution grid," in 3rd European Ele-Drive Transportation Conference, 2008, March 2008. [Online]. Available: http://www.esat.kuleuven.be/electa/publications/fulltexts/pub 1767.pdf

[10] J. Taylor, A. Maitra, M. Alexander, D. Brooks, and M. Duvall, "Evaluation of the impact of plug-in electric vehicle loading on distribution system operations," in Power \& Energy Society General Meeting, 2009. PES '09. IEEE, July 2009.

[11] NEPLAN, Jan. 2010. [Online]. Available: http://www.neplan.ch/

[12] P. N. . SINCAL, Jan. 2010.2 [Online]. Available: http://www.energy.siemens.com/hq/en/services/powertransmission-distribution/power-technologies-international/

[13] NS-2, Jan. 2010. [Online]. Available: http://www.isi.edu/nsnam/ns/

[14] OPNET, Jan. 2010. [Online]. Available: http://www.opnet.com/

[15] QUALNET, Jan. 2010. [Online]. Available: http://www.scalablenetworks.com/

[16] OMNeT++, "The omnet++ simulator," Jan. 2010. [Online]. Available: http://www.omnetpp.org/

[17] INET, "The inet simulation framework," Jan. 2010. [Online]. Available: http://inet.omnetpp.org/

[18] D. Hammerstrom, R. Ambrosio, T. Carlon, J. DeSteese, G. Horst, and R. Kajfasz, "Pacific northwest gridwise testbed demonstration projects, part i and ii," in Pacific Northwest National Laboratory, 2007.

[19] VREG, "Flemish regulation entity for the electricity and gas market," Jan. 2010. [Online]. Available: http://www.vreg.be/ 